\title{
Implementación de piscinas naturales: una alternativa de mejora para los efectos en la vía respiratoria por exposición a piscinas cloradas
}

\section{Implementation of natural pools: An alternative of improvement for effects in airway by exposure to chlorinated pools}

\author{
Carla Paola Sánchez-Ríos, ${ }^{*}$ Mario Trejo-Perea, ${ }^{\ddagger}$ Luisa Daniela Sánchez-Ríos, ${ }^{\ddagger}$ María Fernanda Sánchez-Ríos ${ }^{\ddagger}$
}

*Instituto Nacional de Enfermedades Respiratorias Ismael Cosío Villegas;

`Universidad Autónoma de Querétaro, Querétaro, México.

\begin{abstract}
RESUMEN. Los subproductos de desinfección son el resultado de la reacción de desinfectantes (como el cloro) con materia orgánica natural y están presentes en el agua de las piscinas y en fuentes residenciales como el agua potable, la ducha y el sanitario. Existen estudios que han mostrado efectos en la salud por la exposición a subproductos de desinfección en la disfunción de la vía aérea y otras complicaciones respiratorias. Se ha documentado que estos productos pueden irritar tanto el tracto respiratorio superior como inferior e inducir síntomas respiratorios diversos, particularmente en niños y nadadores de alto nivel. Una piscina natural (también llamada piscina ecológica, piscina naturalizada, biopiscina) es una piscina en la que para lograr la calidad del agua necesaria, se utiliza un sistema de depuración natural en lugar de productos químicos. Cada vez más la evidencia sugiere que puede ser una alternativa a las piscinas cloradas para eliminar la necesidad de tratamiento químico y gastos en procesos mecánicos y eléctricos, pues minimiza los requisitos de bombeo, y disminuye el posible efecto irritativo en la vía aérea.
\end{abstract}

Palabras clave: Piscina natural, salud respiratoria, subproducto de desinfección.

\section{ANTECEDENTES}

Se sabe que la desinfección del agua potable fue uno de los principales avances en la salud pública del siglo XX. La

Correspondencia:

Dra. Carla Paola Sánchez-Ríos

Instituto Nacional de Enfermedades Respiratorias

Ismael Cosío Villegas, Ciudad de México.

Correo electrónico: pao1144tost@gmail.com

Trabajo recibido: 18-XI-2019; aceptado: 04-III-2020.

Citar como: Sánchez-Ríos CP, Trejo-Perea M, Sánchez-Ríos LD, Sánchez-Ríos MF. Implementación de piscinas naturales: una alternativa de mejora para los efectos en la vía respiratoria por exposición a piscinas cloradas. Neumol Cir Torax. 2020;79(3):197-203. https://dx.doi.org/10.35366/96656
ABSTRACT. Disinfection by-products (DBP) are the result of the reaction of disinfectants (such as chlorine) with natural organic matter and are present in swimming pool water and in residential sources such as drinking water, the shower and the bathroom. There are studies which have shown the health effects of exposure to DBP on airway dysfunction and other respiratory complications. It has been documented that these products can irritate both the upper and lower respiratory tract and induce various respiratory symptoms particularly in children and high level swimmers. A natural pool (also called ecological pools, naturalized pools, bio-pools) is a pool where, to achieve the necessary water quality, a natural purification system is used instead of using chemicals. More and more evidence suggests that they can be an alternative to chlorinated swimming pools, eliminating the need for chemical treatment and expenses in mechanical and electrical processes, minimizing pumping requirements and also, reducing the potential irritating effect to the airway.

Keywords: Natural pool, respiratory health, disinfection byproduct.

implementación de clorar el agua como técnica de tratamiento estándar causó una gran caída en la mortalidad por enfermedades infecciosas en todo el mundo. ${ }^{1} \mathrm{Al}$ igual que en el agua potable, la desinfección del agua de la piscina conduce a la formación indeseable de subproductos de desinfección conocidos como DBP por sus siglas en inglés (disinfection by-products). La evidencia de estudios toxicológicos y epidemiológicos sobre los efectos adversos en la salud de los DBP ha generado desde hace varios años preocupación sobre la seguridad química del agua de las piscinas. Hasta el día de hoy, la cloración representa el tratamiento de desinfección más utilizado en piscinas. ${ }^{2}$

Los DBP son el resultado de la reacción de desinfectantes (como el cloro) con materia orgánica natural (como la saliva, el cabello o la transpiración) y están presentes en el agua de la piscina y en fuentes residenciales como el agua 
potable, la ducha y el sanitario. Los primeros estudios que investigaron la aparición de DBP en piscinas se remontan a 1980. ${ }^{3}$ Desde entonces, se han realizado muchos estudios para examinar la existencia de DBP en diferentes países. ${ }^{4-6}$ Hoy, después de cerca de 40 años, se han identificado más de 600 DBP, incluidos los trihalometanos (THM) y ácidos haloacéticos (HAA), y la Agencia Internacional para la Investigación del Cáncer (IARC) ha clasificado varios potencialmente cancerígenos para los humanos (grupo 2B). ${ }^{7}$

Existen en la literatura diversos estudios de diferente evidencia científica, los cuales han mostrado los efectos en la salud de la exposición a las DBP residenciales identificando una asociación con mayor riesgo de neoplasias, además del desarrollo de disfunción de la vía aérea y otras complicaciones respiratorias con la exposición a largo plazo a piscinas. ${ }^{8,9} \mathrm{Si}$ bien esto se ha observado de manera más consistente entre quienes están expuestos de manera ocupacional como los trabajadores de piscinas y los nadadores profesionales, cualquier persona está en riesgo.10,11

En los últimos años, estudios experimentales en líneas celulares $^{12,13}$ y muestras de sangre ${ }^{14-16}$ han identificado un vínculo entre la genotoxicidad/ mutagenicidad y el estrés oxidativo, especialmente a través de la producción de especies reactivas de oxígeno después de la exposición a diversas formas de DBP. ${ }^{17}$

\section{FORMACIÓN DE LOS SUBPRODUCTOS DE DESINFECCIÓN}

Los DBP se forman a través de la reacción de un desinfectante químico (cloro, dióxido de cloro, cloraminas, ozono) con un precursor orgánico representado por varios componentes de la materia orgánica natural (NOM) y un precursor inorgánico, con mayor frecuencia ciertos iones de haluro. Cualquiera de estos desinfectantes puede transformar (oxidar) una molécula compleja de NOM en restos más simples, que luego son reactivos con cloro o cloraminas adicionales que actúan como un agente de sustitución de halógeno..$^{18}$ Algunos de los desinfectantes también pueden oxidar ciertos iones de haluro, bromuro y yoduro, a bromo y yodo, que son oxidantes menos efectivos, pero que son agentes de sustitución más efectivos que el cloro. ${ }^{19}$ En una mezcla de cloro y bromuro y/o yoduro se forman especies mixtas de DBP que contienen cloro, bromo y/o yodo. Además de la presencia de haluros, otros factores influyentes que afectan la formación de DBP halogenados incluyen la concentración de NOM como carbono orgánico disuelto (DOC), pH, temperatura, concentración de desinfectante y tiempo de reacción..$^{20,21}$

Tanto la cantidad como la composición de NOM son influyentes. Los principales tipos/categorías de NOM incluyen alóctonos (derivados terrestres, dominados por sustancias húmicas) y autóctonos (derivados de microbios, en los que la materia orgánica no húmica se vuelve más dominante). ${ }^{22}$ Una característica principal de la NOM, la absorbancia ultravioleta específica (SUVA), es útil para diferenciar los componentes/precursores de NOM húmicos (SUVA más altos) de los no-húmicos (SUVA más bajos). ${ }^{23}$

En la mayor parte del mundo las piscinas públicas por lo común se desinfectan clorando el agua con hipoclorito de sodio o calcio, gas de cloro o cloroisocianuratos. Estos desinfectantes clorados, denominados «cloro», se agregan al agua para liberar ácido hipocloroso, un oxidante potente que es el biocida activo. Al reaccionar con los compuestos de nitrógeno causados por el sudor, la saliva o la orina de los nadadores, el ácido hipocloroso genera una mezcla compleja de subproductos nocivos, de los cuales los más irritantes son las cloraminas. Las monocloraminas y las dicloraminas $\left(\mathrm{NH}_{2} \mathrm{Cl}\right.$ y $\mathrm{NHCl}_{2}$, respectivamente) son solubles en agua, y la suma de sus concentraciones en agua se llama cloro combinado. Por el contrario, la tricloramina, también llamada tricloruro de nitrógeno $\left(\mathrm{NCl}_{3}\right)$, es un gas insoluble en agua que después de su formación se libera inmediatamente en el aire, dando a las piscinas cubiertas su peculiar olor a «cloro». ${ }^{24}$

\section{EFECTOS EN LA SALUD RESPIRATORIA POR EXPOSICIÓN A DBP}

La actividad física regular se reconoce como una medida efectiva de promoción de la salud. Entre las diversas actividades, una gran parte de la población prefiere la natación que, aunque es generalmente beneficiosa, los datos recientes sugieren que a veces también puede tener efectos perjudiciales por exposición a productos químicos resultantes de la interacción entre el cloro y la materia orgánica. ${ }^{25}$

Se ha documentado que estos productos pueden irritar tanto el tracto respiratorio superior como inferior e inducir síntomas respiratorios diversos, particularmente en niños, socorristas y nadadores de alto nivel. Se han realizado estudios en los que la prevalencia de atopia, rinitis, asma e hiperreactividad de las vías respiratorias se encuentra aumentada en comparación con población en general. ${ }^{26}$

En la última década se ha observado que esta afección de la vía aérea puede estar relacionada con el daño epitelial directo y el aumento de la permeabilidad nasal. Los procesos inflamatorios secundarios y la remodelación de las vías respiratorias condicionan síntomas respiratorios crónicos que precisan de tratamientos y pueden disminuir la calidad de vida de las personas expuestas. ${ }^{27}$

Las investigaciones actuales se han dedicado a estudiar los mecanismos por los cuales se documenta una alta prevalencia de hiperreactividad de las vías respiratorias de nadadores o trabajadores de piscinas cubiertas. Recientemente se ha documentado que los nadadores de piscinas cubiertas expresan conteos de células inflamatorias mayores 
12 horas después de los entrenamientos. ${ }^{28,29}$ También se han realizado estudios que evalúan los efectos de diferentes formaciones cloradas en el estrés oxidativo y el daño pulmonar después de los efectos de la natación. Llana-Belloch et al. estudiaron estos efectos después de la exposición a la natación aeróbica en tres piscinas cubiertas con diferentes características, recolectando muestras biológicas para medir el daño pulmonar (proteínas A y B asociadas con surfactante sérico), parámetros de estrés oxidativo (carbonilación de proteínas plasmáticas y malondialdehído y oxidación de glutatión en sangre total) y valores de esfuerzo (lactato sanguíneo) antes y después del ejercicio. No se encontró resultado con significancia estadística; sin embargo, sí hubo tendencia a diferente expresión de proteínas del surfactante entre ambas poblaciones. ${ }^{30}$

Se ha informado que una hora en una piscina clorada es suficiente para aumentar la permeabilidad del epitelio de las vías respiratorias en nadadores. Además, las vías aéreas de los nadadores muestran evidencias de un estrés oxidativo pulmonar de bajo grado en reposo, que parece aumentar después del entrenamiento a largo plazo en piscinas cubiertas, lo que posiblemente sugiere una reducción en las propiedades antioxidantes a nivel pulmonar. Los insultos repetidos al epitelio y la liberación de mediadores inflamatorios en las vías respiratorias pueden ser responsables de un proceso de reparación deteriorado del epitelio y de un proceso de reparación exagerado de las vías respiratorias en los nadadores. ${ }^{31}$

También se ha documentado que la disfunción de la vía aérea en personas expuestas a DBP puede verse afectada a largo plazo por la alta prevalencia de infecciones bacterianas y virales en el entorno de la piscina cubierta, ya que son factores conocidos que pueden inducir/aumentar la inflamación de las vías respiratorias e incrementar la producción de factores de crecimiento, lo que posiblemente influya en la remodelación de las vías respiratorias a largo plazo. ${ }^{32}$
El entrenamiento intenso de natación en piscinas cloradas cubiertas se asocia a largo plazo con cambios en las vías respiratorias similares a los observados en el asma leve, pero con mayor expresión de mucina. ${ }^{33}$

\section{PISCINAS NATURALES}

\section{Funcionamiento, seguridad y costos}

Una piscina natural (también llamada piscina ecológica, piscina naturalizada, biopiscina) es una piscina en la que para lograr la calidad del agua necesaria, utilizamos un sistema de depuración natural en lugar de productos químicos. En estas piscinas los parámetros de calidad del agua se dividen en físico-químicos: $\mathrm{pH}$ óptimo entre 7.2 y 8.0, pH límite entre 6.0 y 9.0, transparencia con criterio de visibilidad del desagüe del fondo, potencial REDOX entre 250 y 900 mV, turbidez inferior cinco unidades nefelométricas de turbidez (UNF), y en parámetros microbiológicos: ausencia de unidades formadoras de colonias (UFC) de E. coli, Pseudomonas aeruginosa y Legionella spp., entre los principales. Cuando no usamos cloro y productos de desinfección del agua en las piscinas convencionales, al caer hojas, insectos, escamas dérmicas y demás productos de materia orgánica se degradan y se generan partículas de amoníaco y otros compuestos. La acumulación de estos productos alimenta a las algas que crecen y mueren, lo que genera más materias orgánicas y nutrientes en una espiral de generación de biomasa, proceso conocido como eutrofización. Es así como las algas unicelulares hacen que se pierda el primer parámetro de calidad, la transparencia del agua. Sin embargo, éste no es un parámetro que afecte a la salud. En algunas ocasiones la eutrofización continúa hasta que la cantidad de materia orgánica que hay en la piscina es tal que surgen organismos que son perjudiciales para la salud del usuario y que aparecen en condiciones

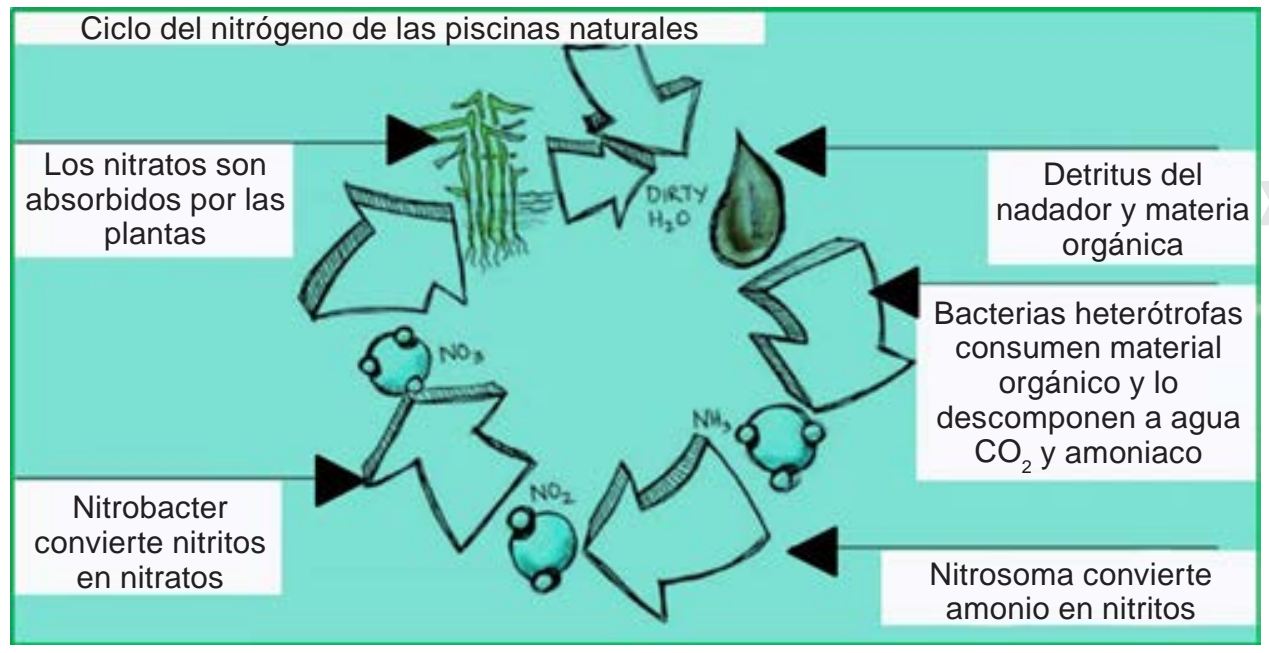

Figura 1:

Modelo de piscina natural. Tomado de: HCMA Architecture + Design. Natural swimming pools. The future of public swimming without chlorine. 2016. ${ }^{34}$ 


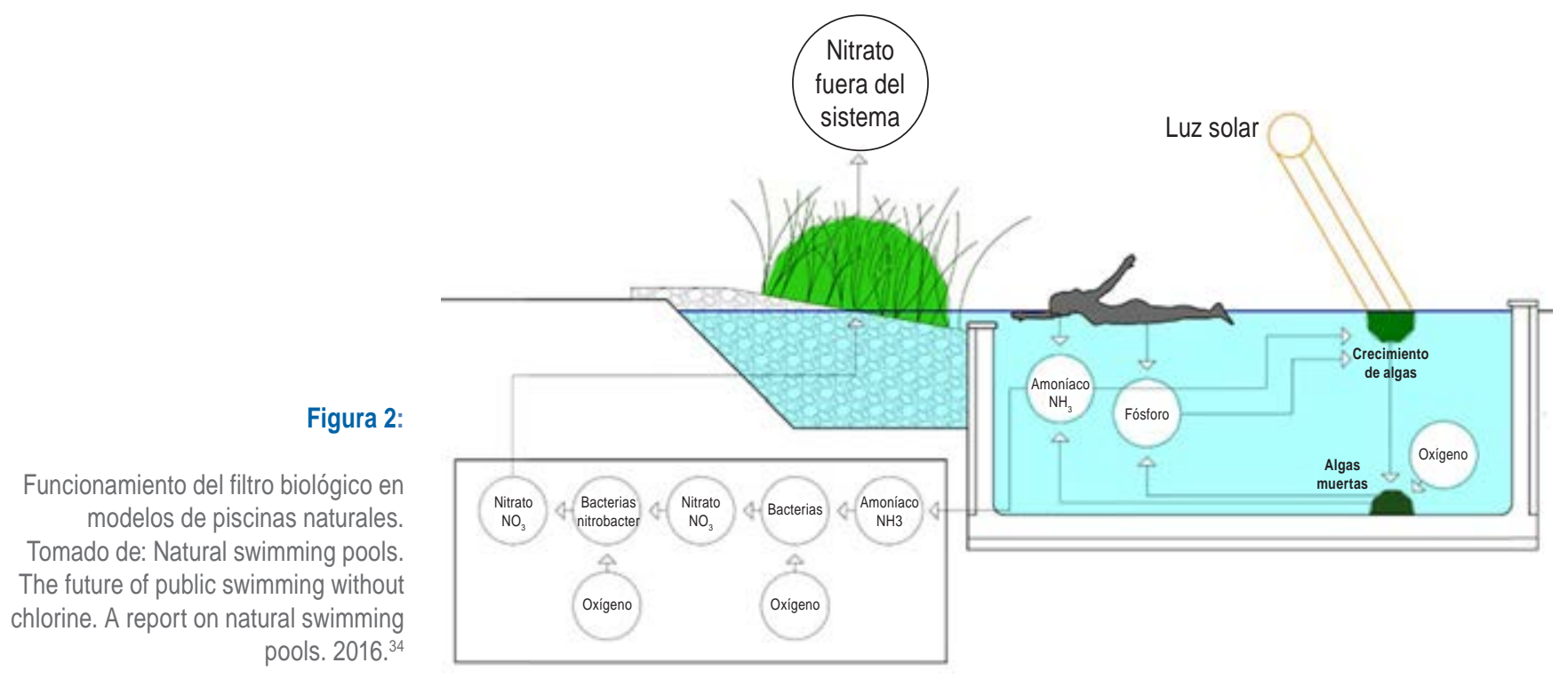

de ausencia de luz solar (falta de transparencia del agua) y acumulaciones de materia orgánica en descomposición (masas de algas, hojas secas, insectos muertos) que generan situaciones anaeróbicas (falta de oxígeno) como son E. coli, Pseudomonas aureoginosa y Legionella spp. En una piscina natural lo que se busca es cortar la espiral de generación de biomasa y eliminar la cadena de procesos y generación de patógenos retirando la materia orgánica del sistema impidiendo que las algas se alimenten de ella. Las plantas acuáticas son las que realizan esta función de eliminación de la materia orgánica (Figura 1).

Sin embargo, los diferentes tipos de algas presentan diversidad en la eficacia a la hora de absorber los distintos nutrientes del agua, haciéndolo antes que las plantas, por este motivo tenemos que facilitar a las plantas la absorción de nutrientes, esto se logra transformando dichos nutrientes mediante la construcción de un filtro biológico.

La materia orgánica en suspensión en el agua de la piscina genera amoníaco $\left(\mathrm{NH}_{3}\right)$, el amoníaco es un nutriente para las algas, pero no para las plantas superiores, además es tóxico para los peces y las personas. Un filtro biológico transforma el amoníaco $\left(\mathrm{NH}_{3}\right)$ en nitrato $\left(\mathrm{NO}_{3}\right)$ mediante la aportación de oxígeno. El nitrato es perfectamente asimilable por las plantas superiores que lo absorben y lo eliminan del sistema de forma que no esté disponible para las algas. Este proceso de oxidación del amoníaco lo realizan una serie de bacterias beneficiosas que viven en el filtro biológico: «nitrosomonas» $y$ «nitrobacter», para efectuar dicho proceso necesitan un material poroso donde la bacteria pueda asentarse y crear colonias y conforme más poroso sea el material, más superficie de asentamiento tendrán las bacterias y el filtro biológico será más eficaz (Figura 2). Una vez trasformado el amoníaco en nitrato, se absorbe y retira el nitrato del agua, ésta es la labor que realizan las plantas en lo que se conoce como zona de regeneración. La poda de las plantas y retirada de restos de las mismas es clave para el correcto funcionamiento de la piscina natural. La zona de regeneración puede estar integrada en el filtro biológico en los casos en que éste sea de flujo vertical, horizontal o percolador; también puede estar separada o integrada dentro del vaso de nado, incluso puede ser una isla flotante en la piscina, existen múltiples posibilidades dependiendo del tipo de filtro y de la apariencia estética de la piscina. Es importante que a la hora de seleccionar las especies de la piscina se deben tener en cuenta varios factores como el clima, los tiempos de floración, si las plantas tendrán follaje todo el año o no y el tipo de fauna que vamos a introducir.

A pesar de todo lo mencionado anteriormente y de que una piscina natural en comparación con las piscinas cubiertas requiere procedimientos de prueba para rastrear y garantizar la calidad del agua, se sabe que una piscina natural a diferencia de estas últimas, elimina la necesidad de tratamiento químico y gastos en procesos mecánicos y eléctricos, y minimiza los requisitos de bombeo, ya que el agua se recicla de manera continua y lo más importante, elimina los riesgos para la salud del agua tratada químicamente.

A futuro se busca maximizar la eficiencia diaria del bombeo de agua a través de la evaporación, ya que estas piscinas acumularían una cantidad similar de agua que una piscina convencional, pero usando menos energía por litro de agua bombeada, lo cual impacta en los costos de mantenimiento. ${ }^{34}$

Actualmente, nuestro país se rige por la Norma Oficial Mexicana NOM-245-SSA1-2010, ${ }^{35}$ requisitos sanitarios y 
calidad del agua que deben cumplir las piscinas. Fue creada con la finalidad de prevenir y minimizar riesgos a la salud pública por enfermedades gastrointestinales, de la piel y otras, ocasionadas por ingestión, contacto e inhalación de microorganismos patógenos y sustancias químicas en el agua de piscinas. En esta norma se menciona que es necesario llevar a cabo el control y vigilancia de las condiciones sanitarias de operación y mantenimiento de las instalaciones, así como el monitoreo sistematizado de parámetros fisicoquímicos y de microorganismos que determinan la calidad del agua; sin embargo, son pocas las aportaciones que en términos de salud respiratoria se mencionan. ${ }^{36}$

Debido a la preocupación por el funcionamiento de este tipo de piscinas, ha estado latente la implementación de piscinas naturales. Por lo comentado en la literatura, estas piscinas son seguras a pesar de la forma de funcionamiento. Hay normativas cuyos lineamientos son los estándares internacionales actuales que toman en cuenta lo mencionado por la Organización Mundial de la Salud en la Guía para Entornos Seguros de Agua Recreativa (WHO Guidelines for Safe Recreational Water Environments), siendo el referente internacional de seguridad microbiológica (Tabla 1). En estos lineamientos se hace referencia a los requisitos de calidad del agua que implican ciertas determinaciones microbiológicas, las cuales tienen valores límite que se deben cumplir para considerar que se encuentran dentro de los estándares internacionales para proporcionar un ambiente seguro y propicio a los usuarios. ${ }^{35}$

En relación con los costos, es fundamental conocer el costo de la adaptación (inversión inicial) y saber el tiempo que se tarda en amortizarse dicha inversión. Para el cálculo de la amortización es crucial el bajo costo de la piscina ecológica frente a una tradicional, esta diferencia es la que decidirá el tiempo de amortización. Actualmente hay

Tabla 1: Requisitos de calidad del agua y determinaciones microbiológicas, Organización Mundial de la Salud (OMS).

\begin{tabular}{|c|c|}
\hline Parámetros & Valores límite \\
\hline Bacterias aerobias a $37^{\circ} \mathrm{C}$ & Inferior a 200 colonias $/ \mathrm{mL}$ \\
\hline Coliformes totales & 10 colonias $/ 100 \mathrm{~mL}$ \\
\hline Coliformes fecales & Ausencia en $100 \mathrm{~mL}$ \\
\hline Estreptococos fecales & Ausencia en $100 \mathrm{~mL}$ \\
\hline Clostridios sulfitorreductores & Ausencia en $100 \mathrm{~mL}$ \\
\hline $\begin{array}{c}\text { Algas, larvas de artrópodos u } \\
\text { otros organismos vivos }\end{array}$ & Ausencia en 1 litro \\
\hline Salmonella sp. & Ausencia en 1 litro \\
\hline Staphylococcus aureus & Ausencia en $100 \mathrm{~mL}$ \\
\hline Pseudomonas aeruginosa & Ausencia en $100 \mathrm{~mL}$ \\
\hline
\end{tabular}

Modificado de: Sánchez Rodríguez JJ.37 pocos estudios en relación a esto; no obstante, se sugiere la investigación realizada por Sánchez Rodríguez en 2011 que menciona los siguientes cálculos:

Costo de la adaptación. Presupuesto total .........144 $\mathrm{m}^{2} \times$ $179.77 € / m^{2}=25,886.88 €$

Costos de mantenimiento al mes............1,314.35 €/mes

Costos de mantenimiento anual............15,772.20 €/año

Esto convertido a pesos mexicanos estaría calculado a costos de adaptación en $\$ 545,951.39$ y de mantenimiento anual \$332,633.92.

Por estas razones, la inversión en una gama más diversa de instalaciones acuáticas que utilizan métodos de tratamiento natural, representaría una buena inversión social. La clave de esta diversificación es la aplicabilidad a la natación al aire libre. Se argumenta que tiene muchos beneficios para la natación en interiores, y aunque a grandes rasgos se considera que aún se requieren procedimientos de prueba para rastrear y garantizar la calidad del agua en nuestro país, se limita el tratamiento químico eliminando compras, equipos mecánicos y eléctricos, los requisitos de bombeo se minimizan y el agua se recicla continuamente, eliminando el riesgo de agua tratada químicamente. Una reducción anticipada en los costos de operación y mantenimiento de las piscinas naturales serviría para reducir el costo por recambio de agua para piscinas al aire libre que tradicionalmente son difíciles de mantener y justificar. En este estudio se identificó que en Cataluña, España en menos de dos años de implementación de piscinas naturales podría tener: 15,786,720 litros menos al año de productos químicos que dañan el medioambiente y a los usuarios, con un ahorro de 4,616,000 $\mathrm{m}^{3}$ al año de agua potable y un ahorro de 72,804,475.20 € anuales de inversión para el funcionamiento de piscinas cloradas. ${ }^{37}$

Los costos aproximados en México para el mantenimiento de una piscina de 50,000 L, sólo en productos químicos y servicios de mantenimiento con visita semanal son de cerca de $\$ 40,000$ a $\$ 50,000$ anuales sin contar el resto de los factores que se incluyen en el cálculo de costos (cantidad de agua, condiciones climáticas, materia orgánica que pueda entrar a la piscina, uso y aseo según el número de personas que la utilizan, condiciones de filtrado y equipo de filtrado).

\section{CONCLUSIÓN}

Debido a la dificultad para realizar estudios de nivel de evidencia comparables con los de un metaanálisis, no existen resultados. Sin embargo, hay gran cantidad de estudios de cohorte, de casos y controles y experimentales que han investigado y concluido que existe una tendencia de posibles efectos nocivos en la salud, en particular en la salud respiratoria, y que han conducido a la mayoría de los 
efectos previamente comentados. Así, consideramos que es un terreno que debe seguirse investigando para tratar de evitar los efectos deletéreos en la vía respiratoria por la exposición a DBP a largo plazo.

\section{REFERENCIAS}

1. Boorman GA. Drinking water disinfection byproducts: review and approach to toxicity evaluation. Environ Health Perspect. 1999;107 Suppl 1(Suppl 1):207-217. doi: 10.1289/ehp.99107s1207.

2. LaKind JS, Richardson SD, Blount BC. The good, the bad, and the volatile: can we have both healthy pools and healthy people? Environ Sci Technol. 2010;44(9):3205-3210.

3. Beech JA, Diaz R, Ordaz C, Palomeque B. Nitrates, chlorates and trihalomethanes in swimming pool water. Am J Public Health 1980;70(1):79-82.

4. Aggazzotti G, Predieri G. Survey of volatile halogenated organics (VHO) in Italy. Levels of VHO in drinking waters, surface waters and swimming pools. Water Res. 1986;20:959-963.

5. Chu H, Nieuwenhuijsen MJ. Distribution and determinants of trihalomethane concentrations in indoor swimming pools. Occup Environ Med. 2002;59(4):243-247.

6. Judd SJ, Jeffrey J. Trihalomethane formation during swimming pool water disinfection using hypobromous and hypochlorous acids. Water Res. 1995;29:1203-1206.

7. IARC. Chlorinated drinking-water; chlorination by-products; some other halogenated compounds; cobalt and cobalt compounds. Lyon, Fran: WHO, IARC; 1997.

8. Lévesque B, Vézina L, Gauvin D, Leroux P. Investigation of quality problems in an indoor swimming pool: a case study. Ann Occup Hyg. 2015;59(8):1085-1089.

9. Villanueva CM, Cordier S, Font-Ribera L, Salas LA, Levallois P. Overview of disinfection by-products and associated health effects. Curr Environ Health Rep. 2015;2(1):107-115.

10. Goodman M, Hays S. Asthma and swimming: a meta-analysis. J Asthma. 2008:45:639-647.

11. Thickett KM, McCoach JS, Gerber JM, Sadhra S, Burge PS. Occupational asthma caused by chloramines in indoor swimming-pool air. Eur Respir J. 2002;19:827-832.

12. Beddowes EJ, Faux SP, Chipman JK. Chloroform, carbon tetrachloride and glutathione depletion induce secondary genotoxicity in liver cells via oxidative stress. Toxicology. 2003;187(2-3):101-115.

13. Yuan J, Wu XJ, Lu WQ, Cheng XL, Chen D, Li XY, et al. Chlorinated river and lake water extract caused oxidative damage, DNA migration and cytotoxicity in human cells. Int J Hyg Environ Health. 2005;208(6):481-488.

14. Du H, Li J, Moe B, McGuigan CF, Shen S, LiXF. Cytotoxicity and oxidative damage induced by halobenzoquinones to T24 bladder cancer cells. Environ Sci Technol. 2013;47(6):2823-2830.

15. Pals JA, Ang JK, Wagner ED, Plewa MJ. Biological mechanism for the toxicity of haloacetic acid drinking water disinfection byproducts. Environ Sci Technol. 2011;45(13):5791-5797.

16. Pals J, Attene-Ramos MS, Xia M, Wagner ED, Plewa MJ. Human cell toxicogenomic analysis linking reactive oxygen species to the toxicity of monohaloacetic acid drinking water disinfection byproducts. Environ Sci Technol. 2013;47:12514-12523.

17. Kogevinas M, Villanueva CM, Font-Ribera L, Liviac D, Bustamante $M$, Espinoza $F$, et al. Genotoxic effects in swimmers exposed to disinfection by-products in indoor swimming pools. Environ Health Perspect. 2010;118(11):1531-1537.

18. Backer LC, Lan Q, Blount BC, Nuckols JR, Branch R, Lyu CW, et al. Exogenous and endogenous determinants of blood trihalomethane levels after showering. Environ Health Perspect. 2008;116(1):57-63.

19. Gary GL. Improved exposure assessment on existing cancer studies. Denver, CO: AWWA Research Foundation and American Water Works Association. 2005. p. 88.

20. Whitaker HJ, Nieuwenhuijsen MJ, Best NG. The relationship between water concentrations and individual uptake of chloroform: a simulation study. Environ Health Perspect. 2003;111(5):688-694.

21. Karanfil T, Krasner SW, Westerhoff P Xie Y. Avances recientes en la formación de subproductos de desinfección, ocurrencia, control, efectos sobre la salud y regulaciones. ACS Symposium Series. 2008. Vol. 995, pp. 2-19. [14 de noviembre de 2019] Disponible en: https://asu.pure.elsevier.com/en/publications/recent-advances-indisinfection-by-product-formation-occurrence-c

22. Ross MK, Pegram RA. Glutathione transferase theta 1-1dependent metabolism of the water disinfection byproduct bromodichloromethane. Chem Res Toxicol. 2003;16:216-226.

23. Xu X, Weisel CP. Human respiratory uptake of chloroform and haloketones during showering. J Exp Anal Environ Epidemiol. 2004;15:6-16.

24. Zwiener $\mathrm{C}$. Drowning in disinfection byproducts? Assessing swimming pool water. Environ Sci Technol. 2007;41:363-372.

25. Vidarte J, Velez Alvarez C, Cuellar C, Alfonso M. Actividad física: estrategia de promoción de la salud. Hacia la promoción de la salud. 2011;16:202-218.

26. Font-Ribera L, Kogevinas M, Zock JP, Nieuwenhuijsen MJ, Heederik D, Villanueva CM. Swimming pool attendance and risk of asthma and allergic symptoms in children. Eur Respir J. 2009;34(6):1304-1310.

27. Schoefer Y, Zutavern A, Brockow I, Schäfer T, Krämer U, Schaaf B, et al. Health risks of early swimming pool attendance. Int J Hyg Environ Health. 2008;211(3-4):367-373.

28. Bernard A, Carbonnelle S, Michel O, Higuet S, De Burbure C, Buchet JP, et al. Lung hyperpermeability and asthma prevalence in schoolchildren: unexpected associations with the attendance at indoor chlorinated swimming pools. Occup Environ Med. 2003;60(6):385-394.

29. Li JH, Wang ZH, Zhu XJ, Deng ZH, Cai CX, Qu LQ, et al. Health effects from swimming training in chlorinated pools and the corresponding metabolic stress pathways. PLoS One. 2015;10(3):e0119241.

30. Llana-Belloch S, Priego Quesada JI, Pérez-Soriano P, Lucas-Cuevas ÁG, Salvador-Pascual A, Olaso-González G, et al. Disinfection byproducts effect on swimmers oxidative stress and respiratory damage. Eur J Sport Sci. 2016;16(5):609-617.

31. Lomax M. Airway dysfunction in elite swimmers: prevalence, impact, and challenges. Open Access J Sports Med. 2016;7:55-63.

32. Bonadonna L, La Rosa G. A review and update on waterborne viral diseases associated with swimming pools. Int J Environ Res Public Health. 2019;16(2):166.

33. Bougault V, Loubaki L, Joubert P, Turmel J, Couture C, Laviolette M, et al. Airway remodeling and inflammation in competitive swimmers training in indoor chlorinated swimming pools. J Allergy Clin Immunol. 2012;129(2):351-358, 358.e1.

34. HCMA Architecture + Design. Natural swimming pools. The future of public swimming without chlorine. 2016 [18 November 2019] Available in: https://hcma.ca/wp-content/uploads/2016/04/Natural-SwimmingPools-Report_HCMA.pdf 
35. World Health Organization. Guidelines for safe recreational water environments. Volume 1: Coastal and fresh waters. Geneva: World Health Organization. 2003. [26 December 2019] Available in: pps. who.int/iris/bitstream/handle/10665/42591/9241545801.pdf;jsessio nid $=4 F A 44 D D 9520 F 50 A C A 553 D 2 A 90 B 3 F B 513$ ? sequence $=1$

36. NOM-245-SSA1-2010, Requisitos sanitarios y calidad del agua que deben cumplir las albercas. Tomado de: http://dof.gob.mx/nota_detalle. php?codigo=5256066\&fecha=25/06/2012 el 27 diciembre de 2019.
37. Sánchez Rodríguez JJ. Estudio de la implantación de piscinas ecológicas en las piscinas municipales de Cataluña, España [Proyecto final de carrera]. España: Universidad Politécnica de Cataluña; 2011 [17 de diciembre de 2019] Tomado de https://upcommons.upc.edu/ bitstream/handle/2099.1/11554/MEMORIA.pdf

Conflicto de intereses: Los autores declaran no tener conflicto de intereses. 